\title{
Improvement of the Transient Stability of a 14-bus Network Using a Superconducting Fault-Current Limiter SFCL
}

\author{
Salah Belkhiri, Malak Bouroubi, Asma Harrabi \\ Department of Electrical Engineering, Faculty of technology, University of M'Sila, Algeria \\ Corresponding author: Salah Belkhiri (e-mail: salah.belkhiri@univ-msila.dz).
}

\begin{abstract}
This study presents a new strategy for implementing the superconducting fault current limiter SFCL in order not only to limit short - circuit currents but also to improve the stability of electrical networks in the presence of faults. The calculation of the impedance that the limiter must introduce into the test network at the moment of the failure is obtained from a three-dimensional computation code, developed and implemented under MATLAB environment where the formulation in magnetic vector potentials A and scalar potential Electric $\mathrm{V}$ is adopted to solve the electromagnetic problem and the heat diffusion formulation is adopted also to solve the thermal problem. The coupling is ensured by an alternating algorithm and the numerical resolution of the problem is ensured by the method of the finite volumes in its three-dimensional version in order to avoid certain problems of numerical convergence linked to the strongly nonlinear character of the problem to be solved. The modeling of the network tests will be made by PSAT under the environment MATLAB.
\end{abstract}

INDEX TERMS Critical clearing time (CCT), Electromagnetic and thermal phenomena, Finite volume method, High temperature critical, Superconducting faults current limiter (SFCL), Transient stability.

\section{INTRODUCTION}

$\mathbf{T}$ he study of the stability of electrical networks is one of the most important aspects in the analysis of power systems. It determines whether or not the system can find a new operating point after a disturbance [1-2].

In recent years, the problems of stability of power grids have a lot of attention. Several studies and techniques have been directed and developed for the system maintains stability under any disturbance. These disturbances may be of various origin; (small disturbances: as the continuous variations of load, large disturbances such as short circuits [3] and the loss of synchronism of a high-power generator) [4].

The most important aim of stability studies is to find dynamic behavior the main variables that determine the operation of the generators as well as the angle, speed, current, voltage and power [5]. Even with these variables, it is possible to determine the critical time of fault elimination or the stability margin. In other words, the stability aims to answer the following question: what is the maximum time of release of the fault for which the network remains stable? In the evaluation of stability, the critical time of compensation (CCT) is a very important parameter for maintain the stability of the power system. The CCT is maximum duration during which a failure can occur in power system without loss of stability. Elimination of defects time is set randomly. If the Fault Elimination Time (FET) is greater than CCT, the relative angles of the rotor will be shifted, and the system will lose its stability [6].

In this context, several simulation works have been proposed. In some of these works, the behavior of the superconductor is simulated as a vari-impedance [7], [8-9] where the superconducting material changes from nondissipative state characterized by a zero impedance in the rated regime of the network to a very dissipative state characterized by a high impedance in the case of faults that can appear during the operation of the electrical network.

These simple models developed do not satisfactorily reflect the actual behavior of the superconductor in its intermediate state, particularly the flux-flow and flux-creep regimes [10]. For this, other microscopic models have been proposed in order to satisfactorily describe the flux-flow and flux-creep regimes [11-12]. In these models, the Maxwell equations are adopted and coupled to the heat diffusion equation, however the electromagnetic and thermal problems are solved in the case of one-dimensional [13] see bidimensional [14]. These models cannot correctly simulate superconducting current limiters, in particular, superconducting current limiters.

In order to show the contribution of the SFCL on transient stability, the proposed model should be integrated into the transient stability program. In this paper, we present the modeling and improving of the power systems transient stability of multi-machine fourteen buses with the PSAT under 
the MATLAB environment and analysis is performed with the fault located in a bus.

\section{FORMULATION}

Use either SI (MKS) or CGS as primary units. (SI units are To model the magnetic behavior of the presented problem, we adopted the formulation in magnetic vector potentials $\mathrm{A}$ and in electric scalar potential $\mathrm{V}$, this one is described by the formulation below.

$$
\begin{aligned}
& \nabla \times(v \nabla \times \mathrm{A})-\nabla(v \nabla \cdot \mathrm{A})+\sigma(E, T)\left(\frac{\partial \mathrm{A}}{\partial \mathrm{t}}+\nabla V\right)=\mathrm{J}_{\mathrm{s}} \\
& \nabla \cdot\left\{-\sigma(E, T)\left(\frac{\partial \mathrm{A}}{\partial \mathrm{t}}+\nabla V\right)\right\}=0
\end{aligned}
$$

$\boldsymbol{v}$ and $\boldsymbol{\sigma}$ represent respectively the magnetic reluctivity and the electrical conductivity of the superconductor. Concerning the apparent electrical conductivity of the superconducting material, in its non-dissipative state, it is defined by the ratio of $\mathrm{J}$ on $\mathrm{E}$ [15-16], this ratio is deduced from the characteristic E-J of the superconductor given by the relation.

$$
\begin{gathered}
\sigma_{S}(E, T)=\frac{J}{E}=\frac{J_{c}(T)}{E_{c}}\left(\frac{E}{E_{c}}\right)^{\frac{1}{n(T)}-1} \\
\text { With } \quad \\
J_{c}(T)=J_{c 0} \frac{\left(1-\frac{T}{T_{c}}\right)}{\left(1-\frac{T_{0}}{T_{c}}\right)}
\end{gathered}
$$

This relation reflects the superconductors Flux-Flow and Flux-Creep regimes that is to say if the superconductor is in a non-dissipative state, to complete the expression of the electrical conductivity of the superconductor in the dissipative regime. Add an additional term $\sigma_{n}$ which translates the increase in the resistance of the superconductor. Thus, the apparent electrical conductivity of the superconductor is deduced by the relation.

$$
\sigma(E, T)=\sigma_{s}(E, T)+\sigma_{n}(T)
$$

Where $\mathrm{J}_{\mathrm{C}}$ and $\mathrm{E}_{\mathrm{C}}$ respectively represent the density of the critical current and the critical electric field. According to relation (3), the apparent conductivity of the superconductor depends on the electric field $\mathrm{E}$ and the temperature $\mathrm{T}$ reached within the material. The electric field $\mathrm{E}$ will be determined from the resolution of the electromagnetic problem described by the partial differential equation presented by the formulation (1). The temperature will be determined from the resolution of the heat diffusion problem presented by $\rho C_{p}(T) \frac{\partial T}{\partial t}-\nabla \cdot(\kappa(T) \nabla T)=W$

Where $\lambda(T), \rho, C p(T)$ are respectively the thermal conductivity in $(\mathrm{W} / \mathrm{K} / \mathrm{m})$, the density in $\left(\mathrm{Kg} / \mathrm{m}^{3}\right)$ and the specific heat of the material in $(\mathrm{J} / \mathrm{K} / \mathrm{Kg}), \mathrm{W}$ is a power density in $\left(\mathrm{W} / \mathrm{m}^{3}\right)$, it expresses all the losses generated in the superconducting current limiter expressed by

$W=E . J$

In the results of the simulations presented, the thermal and the electrical properties depend on the temperature as mentioned in the equations (1), (2), (3), (4). The Models describing this dependence are presented in [16].

The resolution of the system of equations 1 and 4 solves electromagnetic and thermal problems. These are defined by strongly nonlinear equations. To solve such a problem, several methods have been used mainly finite element method [11-12], it cannot ensure the convergence of the problem to be solved especially during the presence of a superconducting material where have used a power type law to define electrical conductivity (Equation 2). To avoid this type of problem we used the finite volume method in its three-dimensional version. The adopted mesh is of Cartesian or structured type, it consists of elementary volumes of hexahedral or cubic form. For each volume Dp of hexahedral shape, we associate a so-called main node $\mathrm{P}$ and six facets: $\mathrm{e}$ and $\mathrm{w}$ in the direction $\mathrm{x}, \mathrm{n}$ and $\mathrm{s}$ in the direction $\mathrm{y}, \mathrm{t}$ and $\mathrm{b}$ in the $\mathrm{z}$ direction (Fig.1). The neighboring volumes of $\mathrm{Dp}$ are represented by their close neighboring nodes: $\mathrm{E}$ and $\mathrm{W}$ along the $\mathrm{x}, \mathrm{N}$ and $\mathrm{S}$ axis along the $\mathrm{y}, \mathrm{T}$ and $\mathrm{B}$ axis along the $\mathrm{z}$ axis [16].

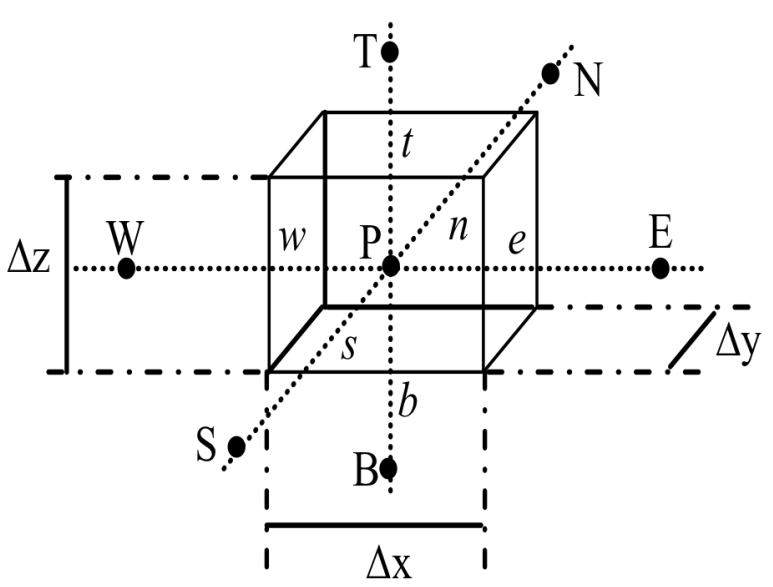

FIGURE 1. Elementary finished volume 


$$
\begin{aligned}
& \iiint_{D_{P}} \nabla \times(v \nabla \times A) d \tau-\iiint_{D_{P}} \nabla(\nu \nabla \cdot A) d \tau+\iiint_{D_{P}} \sigma\left(\frac{\partial \boldsymbol{A}}{\partial t}+\nabla V\right) d \tau=\iiint_{D_{P}} \boldsymbol{J}_{\boldsymbol{s}} d \tau \\
& \iiint_{D_{P}} \nabla \cdot\left\{-\sigma\left(\frac{\partial \boldsymbol{A}}{\partial t}+\nabla V\right)\right\} d \tau=0
\end{aligned}
$$$$
\int_{w}^{e} \int_{s}^{n} \int_{b}^{t} \rho C_{p} \frac{\partial T}{\partial t} d \tau=
$$$$
\int_{w}^{e} \int_{s}^{n} \int_{b}^{t} \frac{\partial}{\partial x}\left(\kappa \frac{\partial T}{\partial x}\right) d \tau+\int_{w}^{e} \int_{s}^{n} \int_{b}^{t} \frac{\partial}{\partial y}\left(\kappa \frac{\partial T}{\partial y}\right) d \tau+\int_{w}^{e} \int_{s}^{n} \int_{b}^{t} \frac{\partial}{\partial z}\left(\kappa \frac{\partial T}{\partial z}\right) d \tau+\int_{w}^{e} \int_{s}^{n} \int_{b}^{t} W d \tau
$$

To calculate the derivative terms in (6) and (7), we consider in our study a linear variation of the magnetic potential and the temperature across the integration facets of the finite volume. After integration, we arrive at a system of algebraic equation below that will be solved by an algebraic method such as the Gausse-Seidel method that will be adopted in our problem. The integral of equations (6) and (7) leads to the following algebraic equations [15-16]:

$$
\begin{aligned}
& c p A_{x}^{P}+\sigma_{p} D_{p} A_{x}^{\bullet P}= \\
& {\left[\sum_{m=e, w \ldots} c_{m} A_{x}^{M}+\sum_{\substack{i=y, z \\
m=e, w \ldots}} q_{m} A_{i}^{M}+\frac{\sigma_{p} D_{p}}{\Delta x_{e}+\Delta x_{w}}\left(V^{E}-V^{W}\right)+J_{s x} D_{p}\right]}
\end{aligned}
$$

$$
c p A_{z}^{P}+\sigma_{p} D_{p} A_{z}^{\bullet P}=
$$$$
\left[\sum_{m=e, w \ldots} c_{m} A_{z}^{M}+\sum_{\substack{i=x, y \\ m=e, w \ldots}} q_{m} A_{i}^{M}+\frac{\sigma_{p} D_{p}}{\Delta z_{t}+\Delta z_{b}}\left(V^{T}-V^{B}\right)+J_{s z} D_{p}\right]
$$

$$
V^{P}=\frac{1}{u_{p}}\left[\sum_{m=e, w \ldots} u_{m} V^{M}+\sum_{\substack{i=x, y, z \\ m=e, n \ldots}} c v_{m} A_{i}^{\bullet M}\right]
$$

$[C] \mid \dot{T}]+[K][T]=[Q]$

The electromagnetic and thermal coupling is ensured by the alternating coupling model.

\section{MODELING OF SFCL}

The model of the superconducting short-circuit current limiter can be represented by an impedance, which depends on current and / or temperature [18-19-20], or a resistance that varies with time [6]. The first model is rather used during a study of the behavior of the limiter strictly speaking (study of the transition from the superconducting state to the resistive state). In our paper, the selection of the optimal location of the limiter is based on the study of the transient stability of the network when a fault occurs. The analysis of the transient stability of a network spread over several seconds, resistance varying over time will be favored to reduce the simulation time (modeling of the simpler behavior). Therefore, the temperature of the superconducting short-circuit current limiter can be as follow (Fig. 2).

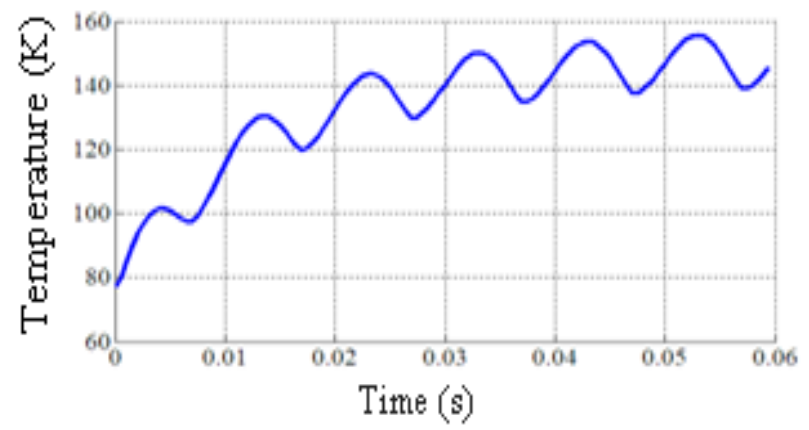

FIGURE 2. Variation of the temperature in the SFCL.

SFCL is a device that limits the fault current by generating impedance when a fault occurs. SFCL installed in series with transmission lines can be just operated during the period from the fault occurrence to the fault clearing, they cannot control the generator disturbances after the clearing of fault.

The equivalent circuit in $\pi$ of the transmission line with SFCL is illustrated in Figure (3). The following admittance matrix of the line can be formed [3-6]:

$Y_{i k}=\frac{1}{\left(r_{i k}+R_{S F C L}\right)+j x_{i k}}$

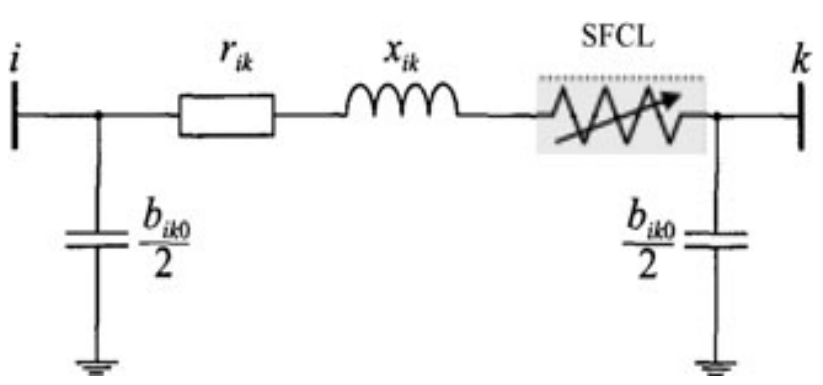

FIGURE 3. Model of SFCL inserted in the transmission line.

The equivalent circuit of generator equipped by SFCL is given by the Figure (4). 
When the resistive SFCL is placed in series with generator, the element of the admittance matrix is given by:

$Y_{i k}=\frac{1}{\left(R_{G}+R_{S F C L}+J X_{d}^{\prime}\right)}$

So after inserting the SFCL into the network, there will be a significant change in the admittance matrix.

We present the simulation results obtained during a fault on a power grid with and without a current limiter using the PSAT software.

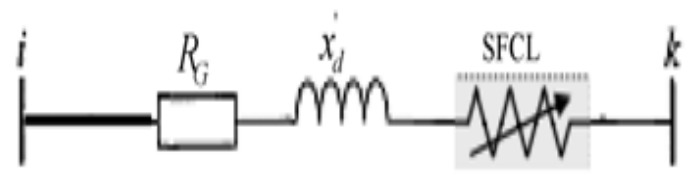

FIGURE 4. Model of SFCL inserted in series with generator.

For the implementation of this type of limiter, several important parameters come into play in this case such as: 1 . The value of the impedance introduced by the limiter in the event of a short circuit; 2 . The optimal position of the limiter in the network; 3. the time of elimination of defects (slow or fast). The proposed method focuses more particularly on points 1 and 3. Various studies have been carried out on the influence of a limiter in a distribution network but the majority has focused on the analysis of a single radial network (a machine, a transmission line, an infinite bus) [21]. In this paper, we propose to extend the study to a network of several alternators that to 14 Bus.

Using the self and transfer admittance, from our code we confirm that the limiting impedance value is $0.13 \mathrm{p} . \mathrm{u}$ and the fault detection time and starting time of limiting impedance are $4 \mathrm{sec}$ and $4.25 \mathrm{sec}$, respectively. Namely, SFCL starts to operate at $0.102 \mathrm{sec}$ [6], and then the limiting impedance increases linearly from $0.0 \mathrm{pu}$ to $0.13 \mathrm{p} . \mathrm{u}$ within $1 \mathrm{msec}$. Although the effect of enhancement of transient stability is changed depend on the limiting resistance value, 0.13p.u is the most effective value on the transient stability enhancement, which is determined based on the results of simulation using various limiting impedance values.

\section{MODELING OF SYSTEM TESTS}

The system used is an IEEE 14 bus system with two generators (G1 and G5) installed at nodes 1 and 2, three synchronous compensating generators (G3, G4 and G2) installed at nodes 3, 6 and 8 . The generators are considered with the limitation on reactive power sets, twenty transmission lines, eleven static charges and four transformers is shown in Figure (5). The base MVA is equal to 100 and the frequency of the system to 60 Hz. the system data are given in the base of IEEE. The default is occurring in bus 4 . The complete system is modeled in PSAT (Power System Analysis Toolbox). The modification of the elements of the admittance matrix is adapted in this work.
The admittance matrix method is based on changing the standard elements (Yij) in coordination with the new model to be integrated. For this network, the admittance matrix $\mathrm{Y}$ is given as follows:

$$
\left[\begin{array}{c}
\mathrm{I}_{1} \\
\mathrm{I}_{2} \\
\mathrm{I}_{3} \\
\mathrm{I}_{4} \\
\cdot \\
\cdot \\
\cdot \\
\mathrm{I}_{14}
\end{array}\right]=\left[\begin{array}{ccccc}
\mathrm{Y}_{11} & \mathrm{Y}_{12} & \mathrm{Y}_{13} & \ldots \ldots \mathrm{Y}_{114} \\
\mathrm{Y}_{21} & \mathrm{Y}_{22} & \mathrm{Y}_{23} & \ldots . . \mathrm{Y}_{214} \\
\mathrm{Y}_{31} & \mathrm{Y}_{32} & \mathrm{Y}_{33} & \ldots . . \mathrm{Y}_{314} \\
\mathrm{Y}_{41} & \mathrm{Y}_{42} & \mathrm{Y}_{43} & \ldots . . \mathrm{Y}_{414} \\
\cdot & \cdot & \cdot & \ldots \ldots \ldots \ldots . \\
\cdot & \cdot & \cdot & \ldots \ldots \ldots \ldots \\
\cdot & \cdot & \cdot & . \\
\mathrm{Y}_{141} & \mathrm{Y}_{142} & \mathrm{Y}_{142} & \ldots & \mathrm{Y}_{1414}
\end{array}\right]\left[\begin{array}{c}
\mathrm{V}_{1} \\
\mathrm{~V}_{2} \\
\mathrm{~V}_{3} \\
\mathrm{~V}_{4} \\
\cdot \\
\cdot \\
\cdot \\
\mathrm{V}_{14}
\end{array}\right]
$$

The dynamics of the machine can be represented by the following differential equations [22]:

$$
\begin{aligned}
& \frac{d \delta}{d t}=w-2 . \pi . f \\
& \frac{d w}{d t}=\frac{d^{2} \delta}{d t^{2}}=\frac{\pi \cdot f}{H}\left(P_{m}-P_{e}\right)
\end{aligned}
$$

The generator electric power output for each machine is computed by following equation.

$P_{e i}=E_{i}^{2} Y_{i i} \cos \left(\theta_{i i}\right)+\sum_{j=1, j \neq i}^{n} E_{i} E_{J} Y_{i j} \cos \left(\delta_{i}-\delta_{j}-\theta_{i j}\right)$

Where

$$
\begin{gathered}
Y_{i j}=\left(Y_{i j}<\theta_{i j}\right)=G_{i j}+j B_{i j} \\
Y_{i i}=\left(Y_{i i}<\theta_{i}\right)=G_{i i}+j B_{i i}
\end{gathered}
$$

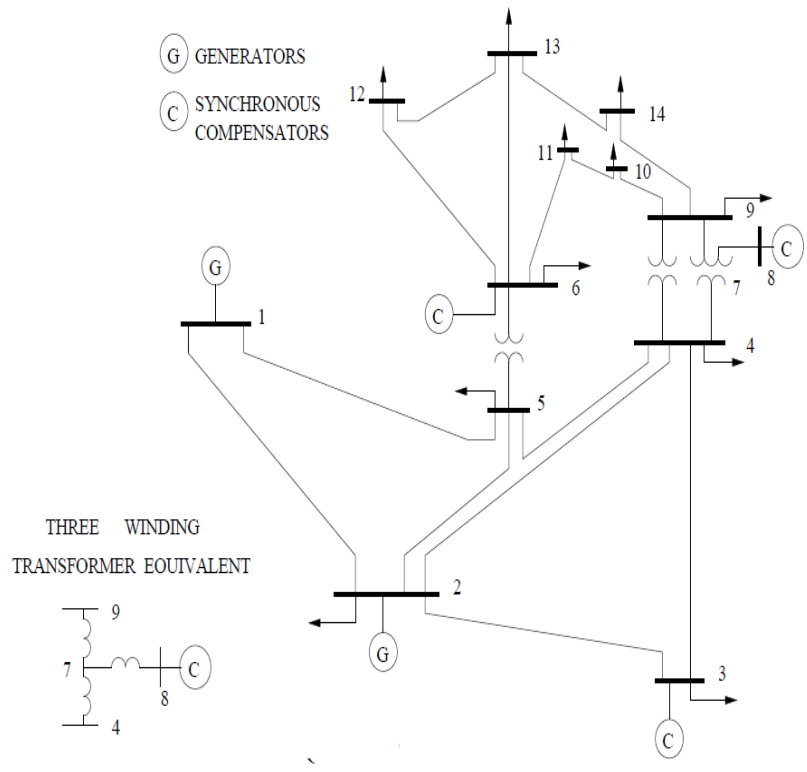

FIGURE 5. IEEE-14 bus test systems. 


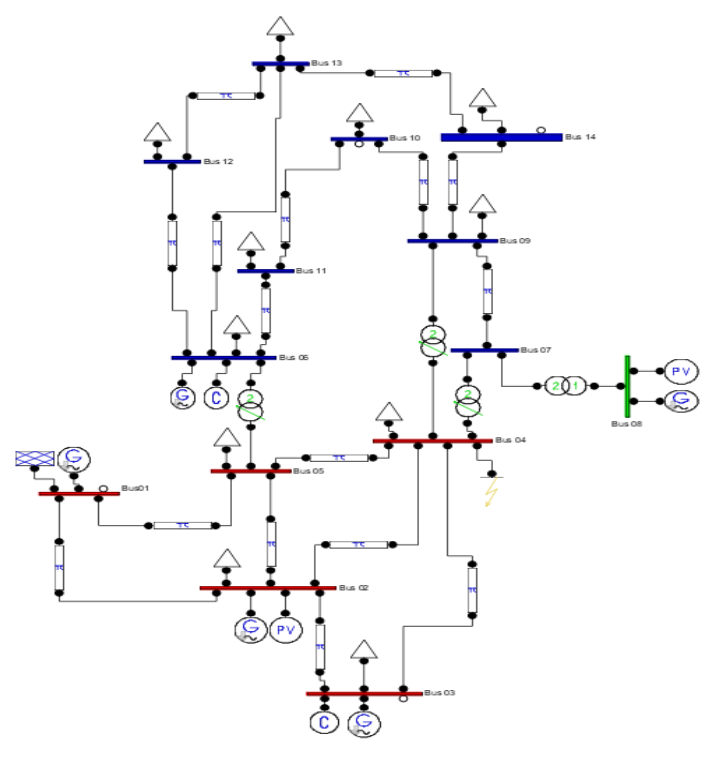

FIGURE 6. PSAT model of IEEE-14 bus test systems.

To analyze the presented method, two SFCL locations (serially with generator and in series with the transmission line) were selected in the test network. We consider that all types of faults can appear in these locations and that each location is suitable for a superconducting current limiter.

The first step of this method consists in presenting the results of simulations (in case the superconducting current limiter is not introduced into the network) for a three - phase short - circuit located in several points of the tested network. For each given location, all of the simulation results were given, which allowed us to obtain the network behavior, in terms of transient stability. The next step is to study the network, always in terms of dynamic behavior, but integrating a superconducting current limiter at the same location where the fault is created (for several locations; generation PV bus, charge PQ bus, slack or swing bus and transmission line). The results thus obtained allow us to evaluate the stability index in the presence of the limiter.

\section{SYSTEM STUDY AND RESULTS ANALYSIS}

\section{A. TRANSIENT STABILITY ASSESSMENT WITH SFCL IN SERIES WITH GENERATOR}

To study the efficiency of and demonstrate the interaction between the SFCL and transient stability of power system, we take the IEEE 14-bus system in the case of three- phase short circuit fault in the several buses.

In this work, the optimal value of the CCT is determined by trial and error. For this, several values of the default duration $\mathrm{Td}$ are previously selected and tested consecutively until the system becomes unstable. The value of this time corresponds to the CCT. To clarify this approach, consider a three-phase fault occurring at time $t=4 \mathrm{~s}$, on different bus bars. The fault persists during a time interval $\mathrm{Td}$ whose value is varied with very small steps in order to obtain the critical value beyond which the stability is no longer ensured, after Td the fault is eliminated, the evaluation the stability of the three periods

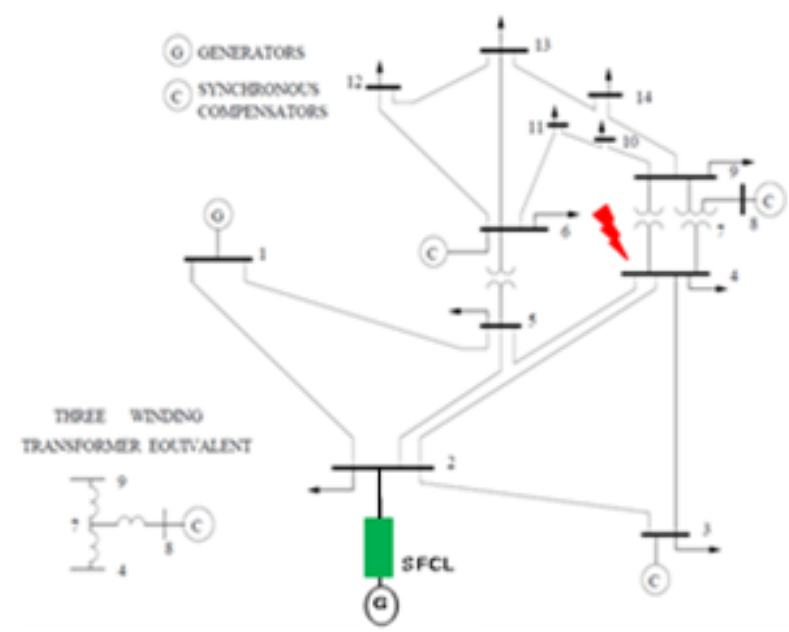

before, during and after default.

FIGURE 7. IEEE-14 bus test systems with SFCL in series with generator № 5 .

The table 1 shows the values of the Critical Clearing Time in Three-phase fault with and without SFCL.

TABLE I. CCT in Three-Phase Fault with SFCL in Series with Generator № 5

\begin{tabular}{llc}
$\begin{array}{c}\text { Fault in } \\
\text { Bus }\end{array}$ & \multicolumn{1}{c}{$\begin{array}{c}\text { CCT(ms) without } \\
\text { SFCL }\end{array}$} & $\begin{array}{c}\text { CCT(ms) with } \\
\text { SFCL }\end{array}$ \\
\hline 01 & 60 & 130 \\
04 & 100 & 200 \\
07 & 260 & 350 \\
10 & 300 & 420 \\
\hline
\end{tabular}

\section{B. TRANSIENT STABILITY ASSESSMENT WITH SFCL IN SERIES WITH TRANSMISSION LINE}

In this study, the SFCL is placed in series with transmission line. The simulation is done in three steps: The prefault is considered for a stable system. Then a symmetrical fault is applied at one transmission line. Simulation of the faulted condition continues until the fault is cleared at $\mathrm{t}=\mathrm{Td}$ (duration of the fault). Then the post-fault system is simulated for a longer time to observe the nature of the transient stability.

The results of CCT after of three phase fault on the different lines are given in Table 2.

Simulations performed on test system indicate that the proposed SFCL can improve transient stability. It can be seen from the results that the system responses are quite satisfied for three-phase short circuit at transmission line and we can say that the fault is more pronounced when it is occurred 
online near the generator bus. Therefore, it is necessary to choose an optimal location for this device; a bad location can reduce the performance of the power system.

TABLE II. CCT in Three-Phase Fault with SFCL in Series with Transmission Line

\begin{tabular}{llll}
\hline $\begin{array}{c}\text { Fault in } \\
\text { bus }\end{array}$ & $\begin{array}{c}\text { SFCL } \\
\text { in line }\end{array}$ & $\begin{array}{c}\text { CCT(ms) } \\
\text { without SFCL }\end{array}$ & $\begin{array}{c}\text { CCT(ms) } \\
\text { with SFCL }\end{array}$ \\
\hline 1 & $1-2$ & 60 & 66 \\
1 & $1-5$ & 60 & 70 \\
4 & $4-7$ & 140 & 146 \\
4 & $4-3$ & 140 & 151 \\
4 & $4-2$ & 140 & 159 \\
4 & $4-5$ & 140 & 151 \\
7 & $7-4$ & 260 & 270 \\
7 & $7-8$ & 260 & 290 \\
7 & $7-9$ & 260 & 320 \\
10 & $10-9$ & 350 & 430 \\
10 & $10-11$ & 350 & 480 \\
\hline
\end{tabular}

A statistical study will be given in the final document so as to choose only one location of the limiter superconductor with a single impedance value because, as we can see in Figure (7), depending on the type of defect studied, several locations of the limiter are possible with several impedance values. We were concerned more particularly in this study by the choice of symmetrical defect by which it is the most unfavorable case for the stability of the networks. With regard to the location of the fault, we chose the slack bus 4 with $\mathrm{Td}=250 \mathrm{~ms}$.

The CCT values obtained by simulation without and with the insertion of the SFCL support us to parameterize the protections in order to isolate the faults and maintain the stability of the system.

It can be noticed that the CCT is a critical factor in judging whether the energy system has a good ability to maintain synchronism and maintain stability during a certain period of disturbance. This critical time proved its importance before the system found the state blackout.

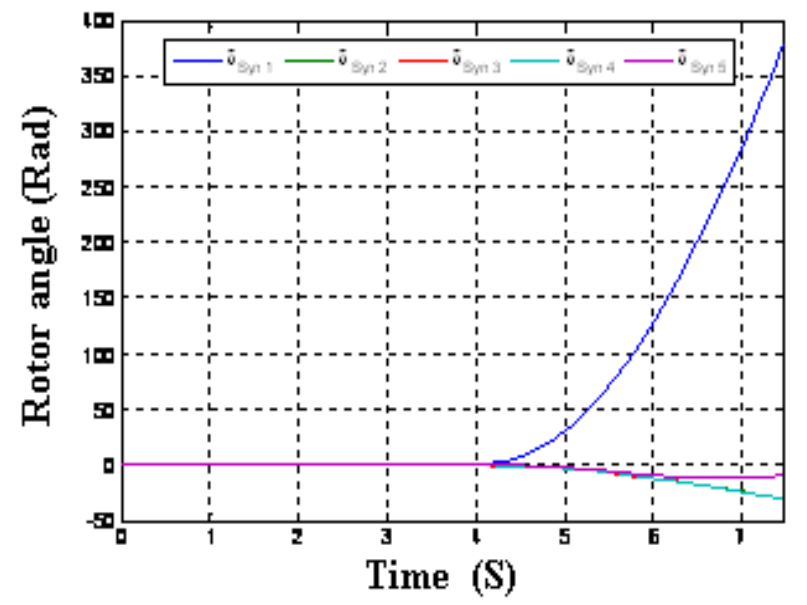

(a) Without SFCL (b) With SFCL

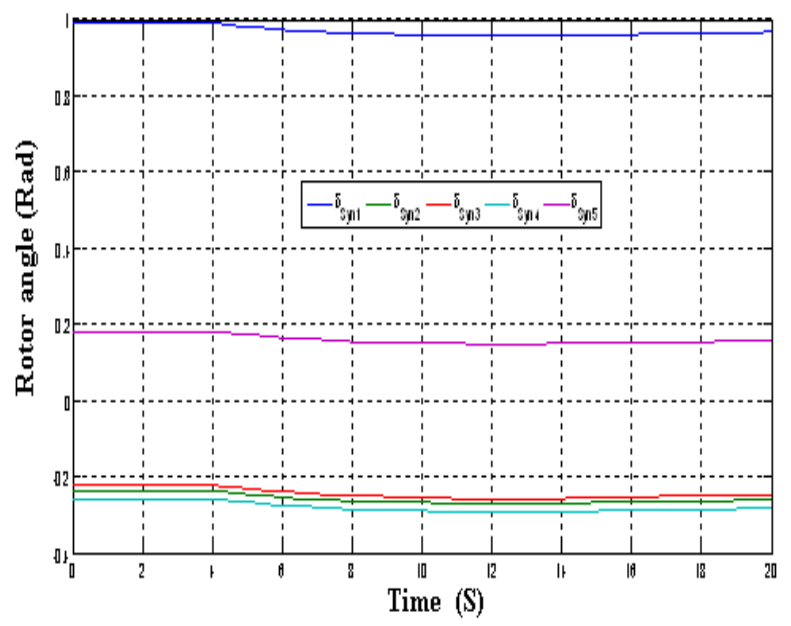

FIGURE 8. Rotor angles (Rad) of 5 generators.

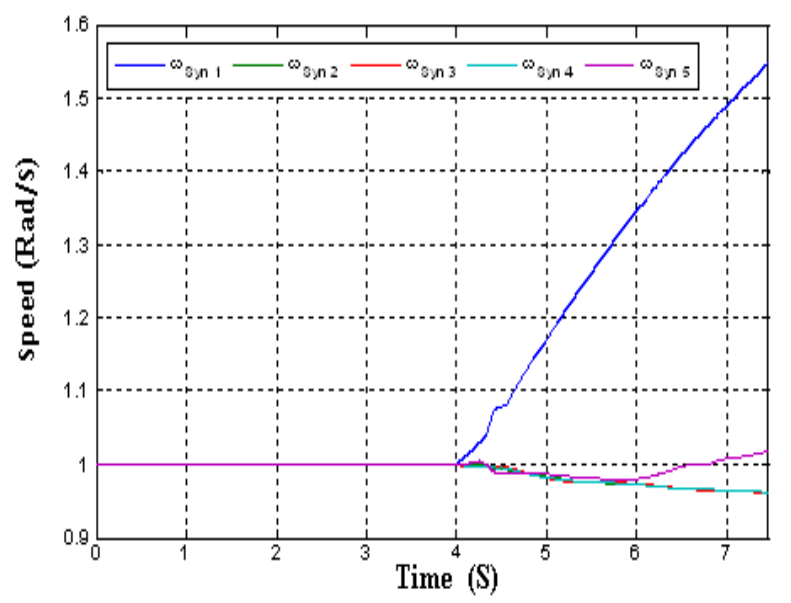

(a) Without SFCL

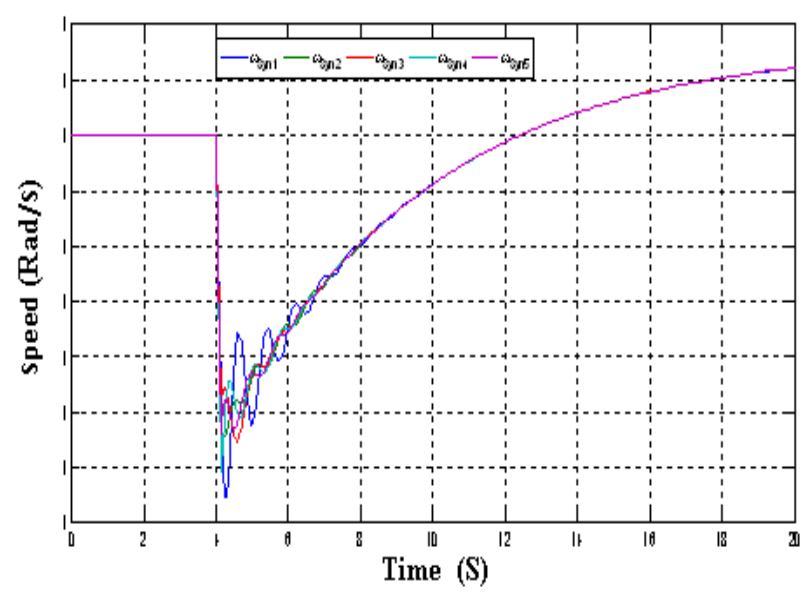

(b) With SFCL 


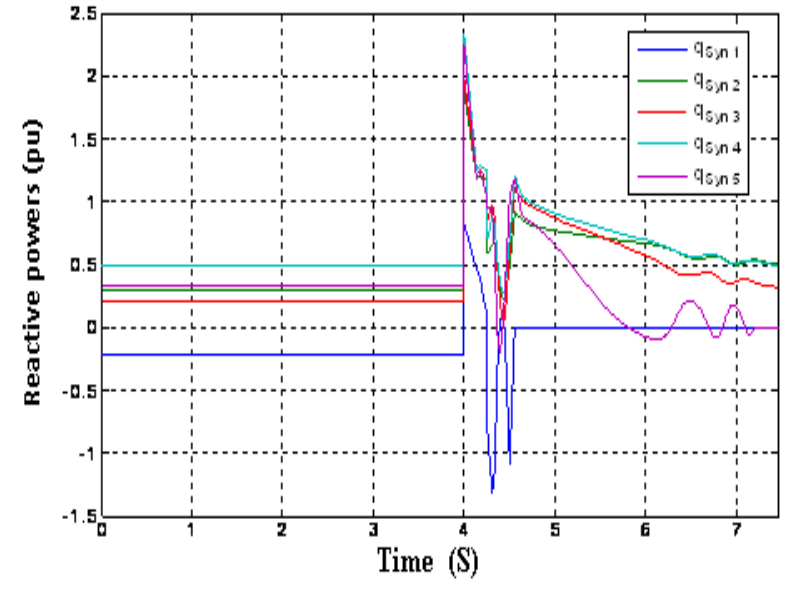

(a) Without SFCL

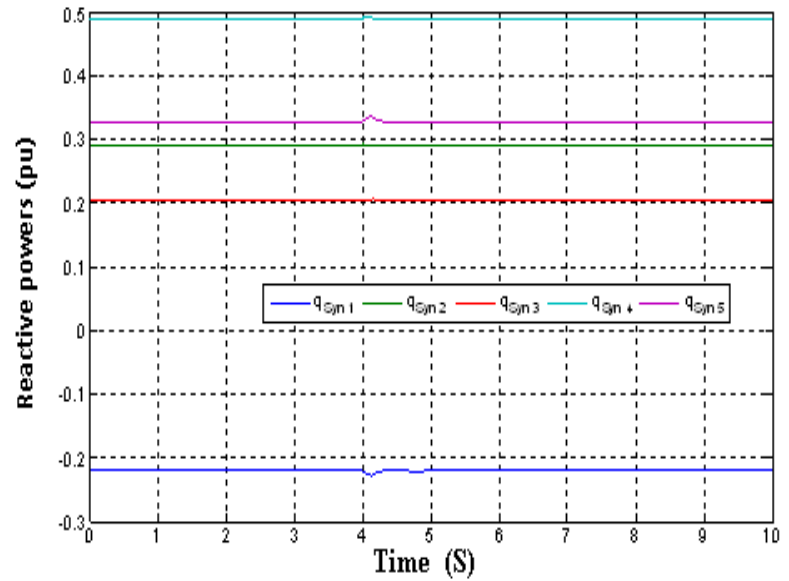

(b) With SFCL

FIGURE 10. Reactive powers (pu) for the 5 generators.

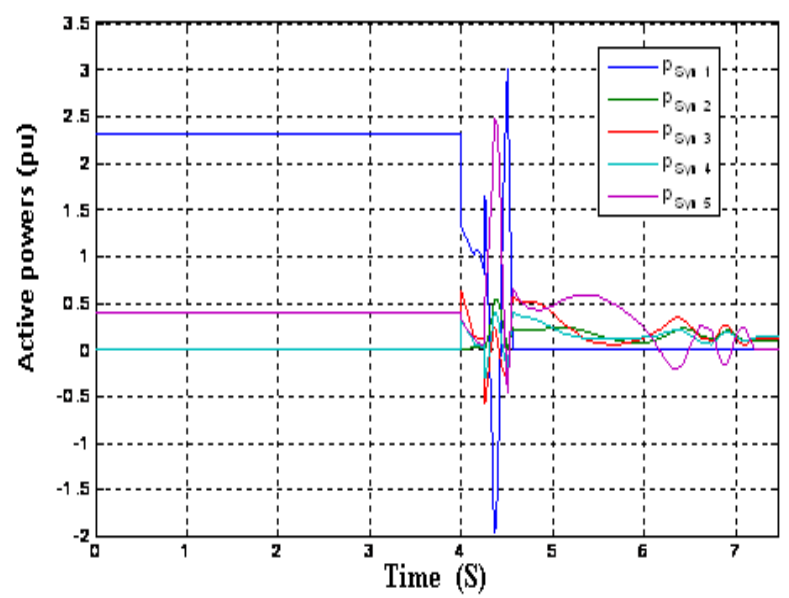

(a) Without SFCL

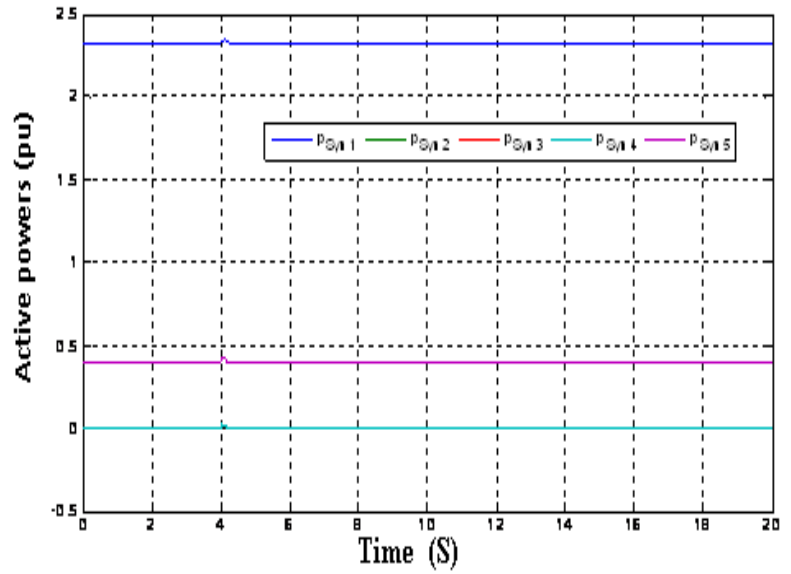

(b) With SFCL

FIGURE 11. Active powers (pu) for the 5 generators.

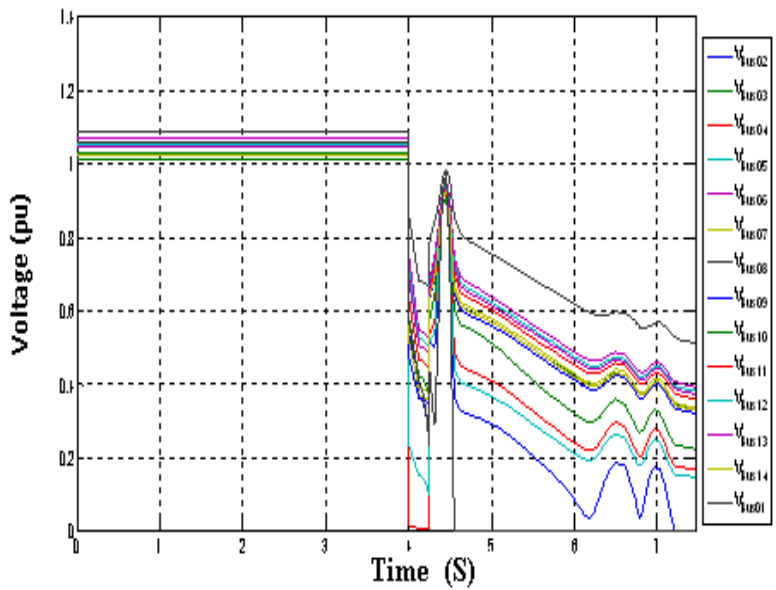

(a) Without SFCL

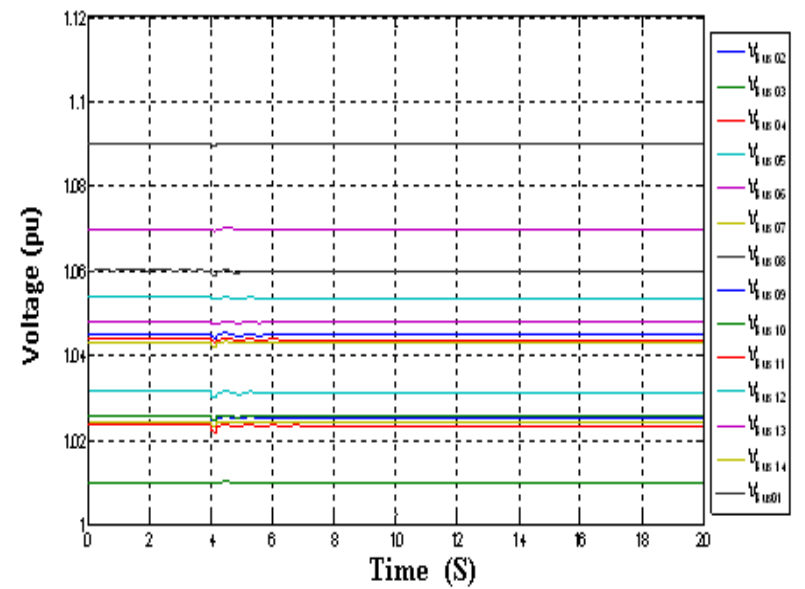

(b) With SFCL

FIGURE 12. Voltage magnitudes. 
Before the insertion of the SFCL, Figures (8(a)) to (12(a)) shows

- For angular velocities there is acceleration for the speed of G1 and G5 and deceleration for the other generators.

- For the powers we see strong decreases of the active power especially for G1 and G5 and an increase for the reactive power but after a few seconds all the powers stiffen towards zero.

- We also find that at $\mathrm{t}=4.4 \mathrm{~s}$ all the voltage decreases rapidly.

After insertion of the SFCL, Figures (8(b)) to (12(b)) confirm that the system remains stable by:

- Small oscillations for the speed of G1 and the system finds the equilibrium point quickly and the same for the angle.

-We also note that the voltages remain stable, the same for the active and reactive powers.

With regard to this assessment and for the defect influence against SFCL, the results presented previously show that the losses in the bus have strongly unbalanced the system. Indeed, for the first case the network goes to the instability, the defect is caused the equality production-consumption reason why in this case the current of defect is greater than the generators cannot provide this requested quantity. And in the 2nd case the network could keep its synchronism and its stability by what the current of defect and small. After this simulation we concluded that the network is unstable for $Z_{\mathrm{SFCL}}=0.0 \mathrm{pu}$ and stable for $Z_{\mathrm{SFCL}}=0.13 \mathrm{pu}$. Then the value of the impedance of the SFCL(Z $\left.Z_{S F C L}\right)$ has a decisive influence on the determination of the value of the fault current more the impedance is important more the fault current is decreased (they are inversely proportional).

\section{CONCLUSION}

In this work, we discussed the influence of short-circuit current on the transient stability of networks. The application was carried out on an IEEE 14 bus network and 5 generators with the PSAT under the MATLAB environment. Three factors are used to study the influence of defect on the stability of the latter. From the analysis, we found that the default location is the most important factor in the extent of defects damage on stability, when the default is near bus generation the percentage of network instability is very big. It is very interesting to note that the default position in relation to the power sources is plays a very important role in the extent of defects damage on stability, as well as the time of fault elimination also plays a very important role import to judge whether the electrical system has a good ability to keep stability during a fault if the protections is delayed, and for the value of the impedance of the SFCL to have a major influence on the limitation of the value of the fault current and by therefore, to find the stability of the system. It should also be noted that the critical clearing time
(CCT) of the fault was decreased as the fault location approached the control panel.

The insertion of the SFCL makes it possible to increase and improves the stability of the system after undergoing a short circuit.

\section{REFERENCES}

[1] P. Kundur, C. Taylor, "Blackout experiences and lessons, best practices for system dynamic performance, and the role of new technologies," IEEE Task Force Report, 2007.

[2] P. Demetriou, M. Asprou. J. Quiros-Tortos, E. Kyriakides, "Dynamic IEEE Test Systems for Transient Analysis," IEEE Systems Journal, vol. PP, no.99, pp.110, 2015.

[3] S. belkhiri, "Modeling a superconducting fault current limiter inserted in a nine-bus electrical network", AMSE. IIETA Journal, Modelling. A, vol. 92, nº. 2-4, pp. 37-42, décembre 2019.

[4] R. K. Mercy, U. R. M. Maheswara, "Transient stability improvement of microgrids by using Resistive type SFCL and series active power filters." Journal EJEE, 2017.

[5] S. Vishwakarma, "Transient stability analysis of two area power system using unified power flow controller, Journal of Advances in Modelling and Analysis," C 73 4 123-127, (2018).

[6] B. Mahdad, K. Srairi, "Application of a combined superconducting fault current limiter and STATCOM to enhancement of power system transient stability," Physica C 495 160-168. 2013.

[7] S. Nemdili, S. Belkhiat, "Electrothermal Modeling of Coated Conductor for a Resistive Superconducting Fault-Current Limiter," J Supercond Nov Magn doi10.1007/s10948-012-1895-4, 2012.

[8] J. Bae, Na and al, "Design and Tests of Prototype Hybrid Superconducting Fault Current Limiter WithFast Switch,” IEEE Trans. Appl. Supercond, vol. 22, no.3, pp. 5602604, 2012.

[9] H. Yamaguchi, and all. "Current Limiting Characteristics of Transformer Type Superconducting Fault Current Limiter With Shunt Impedance," IEEE Trans. Appl. Supercond, vol.17, no. 2, pp. 1919-1922, 2007.

[10] S. Belkhiri, L. Alloui, F. B. Mebarek, "The influence of Geometrical Properties of Bulk Superconductors on Limiting Fault Current in an Electrical Network," ADVANCED ELECTROMAGNETICS, vol. 8, no.4, pp. 136-142, 2019.

[11] W. K. Chan, and al. "Three-Dimensional MicrometerScale Modeling of Quenching in High-AspectRatioYBa2Cu3O7 $-\delta$ Coated Conductor Tapes-Part I: 
Model Development and Validation," IEEE Trans. Appl. Supercond., vol. 20, no. 6, pp. 2370-2379, 2010.

[12] W. K. Chan, and al. "Three-Dimensional MicrometerScale Modeling of Quenching in High-AspectRatioYBa2Cu3O7- $\delta$ Coated Conductor Tapes-Part II: Influence of Geometric and Material Properties and Implications for Conductor Engineering and Magnet Design,” IEEE Trans. Appl. Supercond, vol. 21, no. 6, pp. 2628-2634, 2011.

[13]K. Nam, and al. "Thermal and Electrical Analysis of Coated Conductor Under AC Over-Current," IEEE Trans. Appl. Supercond, vol. 17, no. 2, 1923-1926, 2007.

[14] Casali and al. "Two-Dimensional Anisotropic Model of YBCO Coated Conductors," IEEE Trans. Appl. Supercond, vol. 25, no. 1, pp. 6600112, 2015.

[15] O. Belmont, "Current limitation based on superconducting oxides and realization of a $100 \mathrm{~A}-1 \mathrm{KV}$ device," Ph.D. National Polytechnic Institute of Grenoble - INPG, French. <tel-00763892>, 1999.

[16] L. Alloui, F. Bouillault, and S.M. Mimoune, "Numerical Study of the Influence of flux creep and of Thermal Effect on Dynamic Behaviour of Magnetic Levitation Systems with a high-Tc superconductor using control volume method," EPJ. App. Phys, vol. 37, no. 2, pp. 191-195, 2009.

[17] Y. Yoshida, M. Uesaka, and K. Miya, "Magnetic field and force analysis of high Tc superconductor with flux flow and creep," IEEE Trans. Magn, vol.30, no. 5, pp. 3503-3506, 1994.

[18] H. G. T. Son, H.J. Lee, S.Y. Lee, J.W. Park. "A study on the direct stability analysis of multi-machine power system with resistive SFCL," IEEE Transactions on Applied Superconductivity, vol. 22, no. 3, pp. 5602304-5602304, 2012.

[19]C. Gandioli, P. Tixador, G. Mariani. "Tests and simulations of different YBCO tapes for FCL," IEEE Transactions on Applied Superconductivity, vol. 22, no. 3, pp. 5603104-5603104, 2012.

[20] D. Sousa W. T. B, A. Polasek, F. Silva, R. D. A. Jurelo, R. Andrade, "Simulations and tests of MCPBSCCO2212 superconducting fault current limiters," IEEE Transactions on Applied Superconductivity, vol. 22, no. 2, pp 5600106-5600106. 2012.

[21] G. Didier, J. Leveque, A. Rezzoug, “A novel approach to determine the optimal location of SFCL in electric power grid to improve power system stability," IEEE Transactions on Power Systems, vol. 28, no. 2, pp. 978984, 2013.

[22] H. Shi, X. Zhou, "Stability Analysis on Power System with Large Power Source," Energy and Power Engineering, no. 5, pp. 517-521, 2013. 